Commun: Communication et organisation

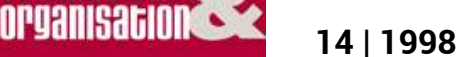

Les formations à la communication

\title{
Enjeux et paradoxes du management par projet
}

\section{Christian Mesnil}

\section{OpenEdition}

Journals

Édition électronique

URL : http://journals.openedition.org/communicationorganisation/2131

DOI : 10.4000/communicationorganisation.2131

ISSN : 1775-3546

\section{Éditeur}

Presses universitaires de Bordeaux

\section{Édition imprimée}

Date de publication : 1 novembre 1998

ISSN : 1168-5549

\section{Référence électronique}

Christian Mesnil, «Enjeux et paradoxes du management par projet », Communication et organisation [En ligne], 14 | 1998, mis en ligne le 26 mars 2012, consulté le 19 avril 2019. URL : http:// journals.openedition.org/communicationorganisation/2131; DOI : 10.4000/ communicationorganisation.2131

Ce document a été généré automatiquement le 19 avril 2019

(c) Presses universitaires de Bordeaux 


\title{
Enjeux et paradoxes du management par projet
}

\author{
Christian Mesnil
}

1 Projet éducatif, pédagogique, politique, professionnel, technique, personnel, contractuel, participatif, collectif, communautaire, d'établissement, de vie, de développement ; avant projet, micro ou mini projet, projet action, animation, pédagogie de projet, etc.

2 Ce relevé non exhaustif nous incite à penser qu'il serait intéressant d'interroger les lexicologues à propos de l'augmentation de la fréquence d'utilisation du mot projet, ainsi que les lexicographes sur la nécessité parallèle d'y adjoindre un second terme qui tente d'en préciser le sens. Faut-il n'y voir que des expressions à la mode, connotées positivement surtout lorsqu'elles sont associées à des termes qui ne laissent personne indifférent comme potentialité, initiative, solidarité?

3 Le terme projet semble en tout cas un terme clé de ces trente dernières années, foisonnant et ambigu.

4 - Terme clé d'une époque qui se dit en crise, en manque de repères, sans projet avec un passé qui n'est plus un sol où s'enraciner et un présent qui s'épuise dans l'éphémère, la consommation ou le virtuel.

5 - Terme d'autant plus foisonnant, décliné sur d'autant plus de registres que l'avenir est porteur d'incertitudes.

6 - Terme ambigu enfin parce que riche de paradoxes, porteur d'espérances et d'inquiétudes renouvelées.

\section{Une société qui ne fait plus de projets?}

7 Que le lecteur me pardonne de commencer cette réflexion par le rappel de ce qui devient presque un lieu commun et qui a pour conséquence un état d'esprit maintes fois identifié : la crise de "sens» d'une société qui donne l'impression à ses observateurs d'avoir perdu ses repères. «On vient d'une époque guerrière, frontale, idéologique, ce qui 
nous permettait de nous adosser à des convictions, à des vérités que l'on tentait d'appliquer sur l'histoire, sur la réalité. Maintenant nous sommes à un moment de mutation, de passage, de creux de vague. Nous sommes revenus déçus de cette grande époque où nous pensions facilement que la raison pourrait transformer la réalité. Et aujourd'hui nous voilà renvoyés à notre vulnérabilité. Nous nous découvrons incapables de transformer les choses. Elles nous échappent " peut-on lire dans l'hebdomadaire Réforme du 15 juin 1996 sous la plume de Remy Hebding.

De ce fait les mots sont de plus en plus suspects. La célèbre répartie d'un ancien ministre de l'intérieur: «Les promesses n'engagent que ceux qui les écoutent» illustre bien le climat présent: méfiance à l'égard des paroles des syndicats et des grands partis politiques et désillusion vis à vis des idéologies (avec des ouvrages comme le Livre noir du communisme par exemple).

9 Fondées ou non, les représentations dominantes actuelles mettent en perspective les langues de bois, la consommation déshumanisée, l'espoir qui s'essouffle, le ressort cassé, la société molle dans laquelle nous végétons.

10 Le scepticisme ambiant semble ainsi entraîner une réticence vis-à-vis de toute réforme, une méfiance vis-à-vis de tout projet caractérisée principalement par le retour au passé ou le repli sur soi.

\section{Le retour au passé}

11 Les commémorations en tout genre se succèdent : bicentenaire de la révolution française, baptême de Clovis, édit de Nantes, abolition de l'esclavage... Le dossier $n^{\circ} 7$ de la revue Communication \& Organisation s'intitulait L'entreprises et ses mémoires. Parler du passé de la nation, chercher à restaurer une part de la mémoire collective, fouiller dans l'histoire de l'entreprise ne constitueraient-ils pas des symptômes révélant la sclérose d'une société en panne de projets?

12 Dans un article intitulé Harassante histoire, Raphaël Picon évoque la fascination pour les temps anciens et la passion avec laquelle nos contemporains s'adonnent à l'exercice commémoratif. "C'est toujours lorsque l'histoire est devenue fébrile et le futur incertain que l'étendue du passé empiète sur le temps présent, que les plus vieilles dates offrent la meilleure certitude que nous avons été depuis fort longtemps et donc que nous sommes encore là aujourd'hui » (Réforme du 28 septembre 1996).

13 Pourtant ce retour au passé n'est pas que repli et refus d'aller de l'avant. D'une part, toute recherche historique ou commémoration est aussi une action mobilisatrice, fédérative, tournée vers un futur, porteuse de dynamismes qui participent manifestement d'une démarche de projets. D'autre part, le procès Papon, la repentance des évêques de France ou l'opposition aux thèses révisionnistes sont des actes de courage, des actes utiles même lorsqu'ils ne sont posés que symboliquement.

14 En résistant au travail de l'oubli, en luttant pour l'émergence ou la préservation de vérités historiques, l'homme œuvre implicitement pour l'avenir en rendant notre passé moins flou et nos valeurs plus lisibles. Ce paradoxe du passé porteur de projets forts et fortement impliquants qui reconstruisent notre société, se retrouve peut-être également dans une seconde attitude de refus apparent des projets. 


\section{Le repli sur soi dans le présent}

15 paradis artificiels en réactualisant une démarche de maîtrise de soi. Par ailleurs la prise de recul préconisée n'est peut-être pas tant un refus d'agir, de bâtir des projets qu'un refus des slogans, des idéologies et des modes. Or un appel à la lucidité ne semble pas foncièrement opposé à une démarche de projet, au contraire ! 


\section{La démarche de projet}

\section{Quelques fondements}

Avoir un ou des projets semble vital pour tout individu. Vivre au jour le jour, sans but, sans savoir dans quelle direction aller tant sur le plan personnel que professionnel plonge les hommes dans la routine et la monotonie.

Pourtant, bâtir un projet, ce n'est pas se lancer à corps perdu dans n'importe quelle activité pour coûte que coûte rompre l'ennui, la solitude et se donner une raison de vivre ou une bonne conscience. Paradoxalement, aller de l'avant, c'est d'abord prendre le temps de s'arrêter, de faire le point, de réfléchir. Or, jeter un regard lucide sur nousmêmes, notre situation et notre environnement sans nous laisser influencer par les publicités, les réflexes de consommation, la mode ou les idées toutes faites n'est pas si simple...

Construire un projet consiste alors à sortir du rôle passif que la société nous propose. L'employé, l'exécutant, le client, le consommateur, le spectateur ou téléspectateur, l'étudiant que nous sommes devient grâce aux projets qu'il met en œuvre un acteur, créateur, artiste, gestionnaire, inventeur, producteur, organisateur, formateur pour luimême et pour les autres.

Cette autre dimension acquise grâce aux projets conduit inévitablement à la prise de conscience de ses propres limites. Aide-toi, le ciel t'aidera, dit le proverbe. Relever le défi envers soi-même, faire le maximum pour combler ses lacunes, vaincre des peurs ou une timidité, progresser, se dépasser est souvent plus facile à dire qu'à vivre vraiment.

Survient alors une seconde découverte tout aussi incontournable: celle de la solidarité. Dès que la réalisation a débuté, il est bien rare de pouvoir aboutir en solitaire absolu. Il y a (presque ?) toujours nécessité de se tourner vers quelqu'un, de disposer d'un appui, d'une compétence, d'une complémentarité. Il va falloir alors demander de l'aide, faire le premier pas, communiquer, s'organiser en équipe, déléguer, œuvrer collégialement et avec transparence, ce qui nécessite une humilité, une vigilance et des ajustements permanents.

29 Enfin, bâtir un projet, c'est aussi trouver un équilibre entre réalisme et utopie. Certes le bon sens doit prévaloir dans le choix d'un but à sa portée, réalisable et/ou rentable, dans la sélection de moyens "raisonnables» (temps, finances...), dans l'efficacité d'une organisation rationnelle, dans la prise de risques mesurés... Il faut savoir cependant qu'il est impossible de se lancer dans un projet, de se projeter en sachant à l'avance où et dans quel état on va aboutir. Le résultat, les conséquences et les retombées ne sont pas à négliger mais appartiennent au futur et seront à évaluer en leur temps.

S'il n'y a pas une part de rêve, de défi, d'aventure, un moment où on est face à l'inconnu, où le courant vous emporte, un moment où il faut coûte que coûte réagir, faire face, se débrouiller... et réussir, il n'y a pas de projet.

\section{Une tension permanente}

31 Deux objectifs difficilement conciliables semblent finalement en tension permanente dans toute démarche de projet. 
32 L'un est centré sur une performance, un résultat, une innovation, un retour sur investissement meilleur qu'avec une démarche classique. Le projet se doit d'enclencher une dynamique avec risque limité et efficacité garantie particulièrement appréciés dans une société de concurrence effrénée. Pourtant il n'y a pas vraiment de schéma théorique infaillible transposable d'un projet à l'autre pour que «ça » marche. Même s'il est mené par un professionnel, chef ou animateur de projet, nul ne peut garantir son aboutissement. Or ce sont ces retombées opératoires, rationnelles, en termes de nouveauté, d'utilité réelle, de temps gagné, de bénéfices éventuels qui importent.

Le second objectif est centré non sur le résultat mais sur la démarche humaine sousjacente. La richesse la plus précieuse à défendre et à développer dans un projet, c'est le processus lui-même alimenté par la diversité et la complémentarité de ses membres.

Quand dans une usine sont installées des boîtes à idées destinées à tous, les changements obtenus seront peut-être minimes mais chaque salarié est invité à passer d'un statut d'exécutant à un statut d'acteur. Il a ainsi le droit de prendre la parole, d'écrire, d'être écouté et lu. Le projet devient un processus pédagogique comme dans les Instituts Universitaires de Technologie qui leur consacrent trois cents heures sur deux années de formation quelle que soit la spécialité concernée (cf. arrêté de 1994). Les étudiants y découvrent que l'ambition d'un projet grandit avec le degré de modestie des différents participants. Modestie non pas quant au projet commun mais dans les prétentions de chacun à vouloir imposer trop massivement ses idées, ses habitudes. Le chemin devient chemin en marchant. Il s'invente en s'y engageant avec les autres. L'essentiel est autant le résultat que les moyens mis en œuvre, la solidarité, la complémentarité, la communication nécessaires.

Dans notre monde néo-libéral, les projets sont curieusement repris à la fois dans une optique de compétition constante où le meilleur projet en terme d'initiative, d'innovation, de gains l'emporte et dans une optique de solidarité, de formation, de préservation ou de restauration de réseaux humains plus ou moins disloqués, en quête d'identité ou de repère. On est alors moins étonné de retrouver peu ou prou des tensions similaires dans différentes pratiques actuelles de projet que la troisième partie de cette étude tente d'identifier.

\section{Quelques pratiques et questionnements actuels}

\section{Les grands et micro-projets}

Les grands projets interviennent plus souvent dans un contexte de grands travaux ou d'édifices publics célèbres (Opéra Bastille, Bibliothèque Nationale, Grand Louvre...).

Ils ont une valeur de symbole, associés au prestige d'un homme politique soucieux de marquer son époque ou parfois aboutissent à une catastrophe financière médiatisée annuellement par le rapport de la Cour des comptes. Ils ont une tendance à être décriés parce que source d'illusion, disproportion entre les moyens mis en œuvre, le résultat obtenu, et d'autres priorités considérées comme injustement écartées. Ils matérialisent pourtant dans la pierre le plus souvent, une part de rêve que chacun de nous porte en soi.

38 Les micro-projets témoignent d'une démarche presque opposée pour s'attacher à des réalisations modestes, des réponses au coup par coup à des besoins concrets. Ils relèvent d'avantage d'associations, d'ONG et s'intéressent plus au développement local, à 
l'insertion, l'intercuturel, le social. Mais ce pragmatisme n'est pas non plus exempt de dysfonctionnements du type saupoudrage, manque de cohérence entre les projets, non adaptation aux besoins réels ou aux mentalités.

\section{Le préalable à un financement ou subventionnement}

La notion de projet s'est développée également en réaction à la course aux subventions et à l'assistanat déshumanisé. Dans le secteur public en particulier, pour être soutenu financièrement, des établissements scolaires, sanitaires, sociaux, culturels doivent présenter des projets précis, viables, argumentés, qui entrent en compétition les uns avec les autres pour la répartition d'une enveloppe budgétaire non extensible. L'accord et le montant des moyens octroyés dépendent de la solidité d'un dossier présenté et du respect de contraintes administratives. Il faudra ensuite dans certains cas rendre compte de l'action menée. Les décideurs et critères de choix sont plus ou moins identifiables. Quant à l'énergie mis en œuvre pour communiquer autour du projet (relations publiques, lobbying...), elle peut en arriver à primer sur l'action projetée elle-même, sans parler des cas extrêmes où le projet devient prétexte pour « décrocher » un financement.

\section{Un outil pour renforcer la motivation}

Dans les organisations pyramidales où la diffusion des messages stratégiques est lente, le projet peut passer pour un nouvel outil de gestion des ressources humaines destiné à relayer d'autres procédures. Malgré diverses théories préconisées depuis vingt ans (voir par exemple L'entreprise du troisième type d'Hervé Serieyx) le travail reste anonyme et remplaçable. On s'y attache en temps de crise pour protéger sa situation et simultanément on s'en désinvestit affectivement parce qu'on n'obtient plus de reconnaissance ou parce qu'on ne fait plus confiance à la direction et au conseil d'administration. Une étude du centre de communication avancée prévoit vers 2015 un management souple fondé sur l'animation et la persuasion (Le Monde 6 février 1996 article de Marie Béatrice Baudet). Les projets constitueraient-ils les prémices de ce management souple ou sont-ils appelés à une vogue éphémère au même titre que le management participatif, les cercles de qualité, le développement personnel ?

\section{La limitation des risques et la flexibilité}

41 L'intérêt observé actuellement dans les entreprises pour les projets est peut-être lié à une conjoncture économique et sociale particulière. Comme il est difficile de mobiliser le personnel sur un avenir imprévisible, la seule gestion possible paraît de plus en plus ponctuelle, concrète. On agit au coup par coup, à la commande ou l'on anticipe mais de façon prudente, limitée, en bâtissant un projet. Celui-ci nous rapprocherait alors des notions de travail temporaire, de flexibilité voire d'intérim pour diminuer les risques. Dans sa ponctualité, le projet participerait paradoxalement à un manque de projection dans l'avenir, à un refus d'anticiper et de courir l'aventure.

Il contribuerait alors à l'atomisation du travail... et à sa déréglementation. On imagine actuellement des contrats de projets qui pourraient remplacer des contrats de travail. Dans ce cas il faudra choisir entre une rémunération « au » projet (comme on disait jadis 
« à » la pièce ou « à » la tâche) et une rémunération globale de salariés travaillant au coup par coup.

Ne partager ni l'emploi, ni le revenu de façon égalitaire mais en fonction du projet? De l'investissement personnel? Selon le résultat? Articuler de façon complémentaire un travail salarié et un engagement volontaire grâce aux projets? Ces questions lourdes de conséquences mettent en avant un autre paradoxe, celui du chacun pour soi lié à l'efficacité des projets.

\section{Les employeurs multiples}

La mobilité professionnelle nous est présentée depuis longtemps comme inéluctable (dynamisme, adaptabilité, progression de carrière...). Cette mobilité ne consisterait plus seulement à changer d'entreprise mais à mener plusieurs projets de front dans différentes entreprises et avoir plusieurs employeurs simultanément. De là à envisager des actions en "auto" emploi avec des projets personnels "auto" rémunérés (et financés!) pour lesquels on fait soi-même appel à des collaborateurs ou sous-traitants, il n'y a qu'un pas.

Les projets peuvent ainsi contribuer à la redéfinition du travail dans une société pluriactive où chacun aurait à négocier son utilité sociale. Une société dans laquelle les statuts salariaux seraient diversifiés (une fiche de paie pour chaque projet indépendant) pose le défi de l'employabilité permanente. Ce défi n'est pas mince lorsque le seul terme de flexibilité, leitmotiv des pays anglo-saxons, est en France presque tabou.

\section{Une nouvelle répartition du travail}

46 Ce paradoxe existe déjà avec des salariés multicartes ou multi-projets s'épuisant au travail alors que le chômage s'étend pour d'autres. La corrélation entre chômage et déconstruction de la personne comme du corps social a été maintes fois observée dans un monde où le travail est un repère d'identité fondamental. Perdre son emploi, c'est déjà ne plus exister.

47 Si au lieu de décompter un horaire de travail, on identifiait différents projets à mener à bien ? Si à côté d'une problématique de la réduction hebdomadaire du temps de travail se mettait en place un inventaire des projets à assurer et une répartition plus équitable de ces derniers? C'est une utopie parce que certaines tâches mécaniques, répétitives ne deviendront jamais projets. C'est une vue de l'esprit car dans une économie libérale, seuls les «bons » projets menés par des professionnels compétents et efficaces seront acceptés et renouvelés.

C'est sans doute en ayant à l'esprit ces objections majeures que Jeremy Rifking dans son ouvrage $L a$ fin $d u$ travail (Éditions La Découverte) évoque un tiers secteur déjà présent dans la société à côté de l'état et du marché qu'il considère comme appelés à régresser. Selon lui les entreprises coopératives, les associations, le bénévolat constituent un tiers acteur amené à se développer, qui doit fonctionner sur d'autres valeurs que le profit dans toutes les failles de l'économie marchande. Les besoins sont là, les projets ne manquent pas. Il doit exister des moyens de gérer des projets, sources de créativité, tout en les sortant de l'économie du marché, des impératifs de financement et de rentabilité. 


\section{Pour conclure, la notion de projet appelle une réflexion plus vaste sur le travail...} de recul grâce à des actions de type projet s'amplifie et que cette notion de projet nous conduit actuellement à une réflexion plus vaste sur la notion même du travail. Comment répartir les gains de productivité ? Diminuer ou supprimer l'emploi ? Réorganiser sous forme de projets un travail intermittent pour le plus grand nombre? Par quel moyen obtenir de la promotion et se construire un avenir souriant? Augmenter les projets de loisir ? Sur quelle base asseoir sa vie?

Le foisonnement des projets - sans jeu de mots - appelle sans doute un projet nouveau politique, social, économique, philosophique - pour bâtir une société capable de retisser du lien social et de l'optimisme.

\section{RÉSUMÉS}

Le terme "projet» semble de plus en plus apprécié mais comporte des aspects variés voire contradictoires que le présent article tente d'identifier.

Le premier paradoxe, c'est que le mot projet soit si souvent employé dans une société qui se dit en crise et qui a tendance à se retourner vers l'histoire ou à se replier sur elle-même dans le présent en retrouvant parfois une attitude proche du stoïcisme antique. Après avoir rappelé quelques fondements d'une démarche projet qui entraînent une tension permanente entre le processus mis en œuvre et le résultat visé, six pratiques actuelles ou conséquences possibles sont envisagées : les grands et microprojets, le préalable à un financement, l'outil de motivation, la flexibilité, les employeurs multiples et la nouvelle répartition du travail. Finalement les retombées entrevues sont signe d'une responsabilisation individuelle croissante et d'une 
dérégulation qui peut conduite soit à des dérives, soit à une redéfinition de la notion même de travail.

The term "project» seems to be more and more appreciated but it includes various, albeit contradictory, aspects : this article tries and identifies them.

The first paradox is that the word project is used so often in a society which sees itself as being in a state of crisis and which tends to withdraw into its history or to turn in on itself in the present, while sometimes rediscovering and attitude similar to ancient stoicism.

After having first recalled a few fundamentals of a project-oriented approach, fundamentals which bring about a permanent tension between the implementation of the process and the results being aimed at, six present practices or possible consequences are considered : big and micro projects, the prerequisite to financing, the motivation tool, flexibility, multiple employers and the new way of dividing up work.

Last, the spin-offs which can be glimpsed at are the sign of a growing awareness of individual responsability and of a deregulation which can lead to either drifting or redefining the notion of work itself.

INDEX

Mots-clés : projet, fondements, motivation, performance, flexibilité, dérégulation

\section{AUTEUR}

\section{CHRISTIAN MESNIL}

Christian Mesnil est Maître de conférences à l'Université du Littoral - Côte d'Opale. Membre du GREC/O (Université Michel de Montaigne Bordeaux 3) il s'intéresse en particulier à la communication d'entreprise et à l'évaluation de compétences. 